\title{
Intervalos de confianza para coeficiente Omega: Propuesta para el cálculo
}

\section{Confidence Intervals for Omega Coefficient: Proposal for Calculus}

\author{
José Luis Ventura-León*. \\ *Universidad Privada del Norte, Lima, Perú.
}

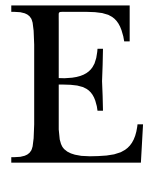

n la actualidad es común el uso de instrumentos de medición para la realización de estudios científicos. Por esa razón, es importante verificar dos propiedades: (a) las evidencias de validez y (b) la fiabilidad de las puntuaciones. Este último impacta de forma directa en la precisión y error de medida (Martínez, Hernández y Hernández, 2014). Razón por la que se sugiere su reporte y cálculo en los estudios científicos.

La fiabilidad es entendida como la capacidad basada en las puntuaciones del instrumento para diferenciar consistentemente entre aquello que tiene mucho de lo que sé está midiendo y poco de ello (Norman, 2014). En su forma clásica, es la proporción de varianza verdadera explicada por los indicadores (Morales, 2013), definición que revela su vinculación con las puntuaciones de un instrumento de medida (Muñiz, 1996) por lo que debe ser reportada para cada estudio en base a la muestra examinada (Wilkinson, 1999).

Los avances en la medición de la fiabilidad han llevado a la creación de diversos coeficientes, entre ellos se encuentra: El coeficiente $\beta$, Coeficiente $\mathrm{H}$ y el Alfa Ordinal un estimador que se adecúa a las demandas de las escalas de salud en donde se utiliza con frecuencia alternativas de respuesta tipo Likert (Zumbo et al., 2007). No obstante, la presente carta trata acerca del coeficiente Omega $(\omega)$ un estimador de fiabilidad relativamente nuevo que es utilizado en modelos factoriales (Ventura-León y Caycho, 2017).

El coeficiente Omega $(\omega)$ es un estimador de consistencia interna basado en las cargas factoriales que indica la proporción de varianza atribuida a la totalidad de la varianza común (McDonald, 1999); es oportuno preferir el $\omega$ por su mayor sensibilidad en comparación con otros estimadores (Zinbarg, Revelle, Yovel y Li, 2005), su resistencia a muestreos en poblaciones heterogéneas y la reducción del riesgo a la sobreestimación de la fiabilidad (Waller, 2008). Asimismo, el $\omega$ no requiere el cumplimiento de tau-equivalencia y la ausencia de errores correlacionados, las cuales son limitaciones del alfa de Cronbach (Dunn et al., 2014) por esa razón puede que el omega supere al alfa y se convierta con el tiempo en una de las opciones preferidas para el cálculo de la fiabilidad (Zinbarg et al, 2005).

$\mathrm{El}$ interés por plantear intervalos de confianza (IC) para el $\omega$, se suscita a partir que la Revista Adicciones ha publicado dos artículos recientemente donde se utiliza este coeficiente (Irles, Morell-Gomis, Laguía, y Moriano, En prensa; Merino-Soto y Blas, En prensa). Por esa razón, el IC resulta un complemento necesario de incluir en estudios venideros en la revista. En ese sentido, el intervalo de confianza (IC) es entendido como rango de valores que sigue una distribución normal y existe la alta probabilidad de encontrar el valor real de una determinada variable (Candia y Caiozzi, 2005). No obstante, es necesario aclarar que el IC se interpreta como la probabilidad de encontrar el valor verdadero en 95 de 100 intervalos producto de extraer muestras aleatorias con las mismas condiciones del estudio (Clark, 2004). En consecuencia, el IC resultante tiene alta probabilidad de contener el valor verdadero de la variable.

El procedimiento de calcular el IC para un coeficiente de fiabilidad, no es situación extraña pues viene desarrollándose en relación al alfa de Cronbach (Domínguez-Lara y Merino, 2015) además de ser recomendada por políticas editoriales (Fan \& Thompson, 2001). No obstante, la obtención de un IC para el $\omega$ plantea el empleo de métodos computacionales. Para tal fin, en la presente carta se expone códigos en R (R Development Core Team, 2007), específicamente de la librería "MBESS" (Kelley \& Lai, 2017) que utiliza el método de bootstrap para la estimación de los

Recibido: Abril 2017; Aceptado: Junio 2017.

Enviar correspondencia a:

José Luis Ventura-León. Av. Tingo María 1122, Breña, Lima.

Email: jventuraleon@gmail.com. 
IC del coeficiente $\omega$. A continuación, se presenta un ejemplo para su estimación:

En primer lugar, se debe instalar y cargar la librería "MBESS" mediante el siguiente código en el programa estadístico R:

install.packages(“MBESS”, dependencies = TRUE)

library(MBESS)

En segundo lugar, se debe activar la función ci.reliability() el mismo que contiene varios argumentos:

ci.reliability (data=felicidad, type="omega", conf.level $=0.95$, interval type="bca", $B=1000$ )

En este ejemplo se está calculando el $\omega$ para una escala de felicidad. Los resultados producto de los cálculos se muestran a continuación (los cálculos suelen demorar unos minutos):

\$est

[1] 0.9098134

$\$$ se

[1] 0.00645999

$\$ c i . l o w e r$

[1] 0.8962767

\$ci.upper

[1] 0.9221084

Tal como se observa en los resultados previamente mostrados, el programa permite calcular el coeficiente $\omega$, error estándar y límite inferior y superior del intervalo de confianza. Cabe mencionar que en el argumento data se puede cargar una matriz de correlaciones y extraer un omega para cada uno de los modelos que se pretenda probar.

Con base en esos resultados el reporte del IC para el $\omega$ es: La escala de felicidad presenta una consistencia interna de .909 por medio del coeficiente omega; asimismo, de acuerdo al nivel de confianza, existe un $95 \%$ probabilidad que el valor verdadero de omega se encuentre en el intervalo resultante $[.896, .922]$.

Finalmente, resulta oportuno brindar un método para la estimación del IC para el $\omega$, debido a su utilización en la revista y su potencial incremento en estudios científicos (Zinbarg et al, 2005). Asimismo, una gran ventaja del método de estimación expuesto en esta carta es lo fácil y amigable de realizar los cálculos; comprendiendo que muchos profesionales son no expertos en estadística. Por esa razón, esta herramienta será útil a investigadores de la Revista Adicciones ayudando al incremento de la precisión de medida en estudios venideros.

\section{Referencias}

Candia, R. y Caiozzi, A. (2005). Intervalos de confianza. Revista médica de Chile, 133, 1111-1115. doi:10.4067/S003498872005000900017.
Clark, M.L. (2004). Los valores P y los intervalos de confianza: ¿en qué confiar? Revista Panamericana de Salud Pública, 15, 293-296. doi:10.1590/S1020-49892004000500001.

Domínguez-Lara, S.A. y Merino, C. (2015). ¿Por qué es importante reportar los intervalos de confianza del coeficiente alfa de Cronbach? Revista Latinoamericana de Ciencias Sociales, Niñez y Juventud, 13, 1326-1328.

Fan, X. y Thompson, B. (2001). Confidence intervals about score reliability coefficients, please: an EPM guidelines editorial. Educational and Psychological Measurement, 61, 517-531. doi:10.1177/0013164401614001.

Kelley, K. y Lai, K. (2017). The MBESS R Package version 4.2.0. Recuperado de https://cran.r-project.org/web/ packages/MBESS/MBESS.pdf

Lloret Irles, D.L., Morell-Gomis, R., Laguía, A. y Moriano, J.A. (2017). Diseño y validación de una escala de intención de consumo de cannabis (CUIQ) para adolescentes. Adicciones. Avance de publicación online. doi: 10.20882 /adicciones.865.

Martínez, A.R., Hernández, J. y Hernández, V. (2014). Psicometría. Madrid: Alianza Editorial.

Merino-Soto, C. y Blas, E.S. (2017). Escala breve de búsqueda de sensaciones (BSSS): estructura latente de las versiones de 8 y 4 ítems en adolescentes peruanos. Adicciones, Avance de publicación on-line. doi:10.20882/ adicciones.842.

Morales, P. (2007). La fiabilidad de los tests y escalas. Recuperado de https://matcris5.files.wordpress.com/2014/04/ fiabilidad-tests-y-escalas-morales-2007.pdf

Muñiz, J. (1996). Teoría clásica de los test. Madrid: Pirámide. Norman, G. (2014). When I say . . reliability. Medical Education, 48, 946-947. doi:10.1111/medu.12511.

R Development Core Team. (2007). R: A language and environment for statistical computing. Viena, Austria: R Foundation for Statistical Computing [programa informático] [en línea] Recuperado de: http:/ / cran.r-project.org/.

Ventura-León, J.L. y Caycho, T. (2017). El coeficiente omega: un método alternativo para la estimación de la fiabilidad [carta al Editor]. Revista Latinoamérica de Ciencias Sociales, Niñez y Juventud, 15, 625-627.

Waller, N.G. (2008). Commingled samples: Aneglected source of bias in reliability analysis. Applied Psychological Measurement, 32, 211-223. doi:10.1177/0146621607300860

Wilkinson, L. (1999). APA Task Force on Statistical Inference. Statistical methods in psychology journals: Guidelines and explanations. American Psychologist, 54, 594604. doi:10.1037/0003-066X.54.8.594.

Zinbarg, R., Revelle, W., Yovel, I. y Li, W. (2005). Cronbach's , Revelle's , and Mcdonald's: Their relations with each other and two alternative conceptualizations of reliability. Psychometrika, 70, 123-133. doi:10.1007/s11336003-0974-7. 